\title{
Al rescate de la economía o cómo hablar con metáforas de asuntos serios ${ }^{1}$
}

\author{
LORENA RIVERA LEÓN \\ Universitat de València \\ Lorena.Rivera@uv.es
}

Resumen: A raíz de la formulación de la teoría cognitiva de la metáfora por parte de Lakoff y Johnson (1980) se descubre que esta figura puede promover el desarrollo de la competencia comunicativa en el aprendizaje de segundas lenguas. Sin embargo, en el ámbito de ELE apenas hay materiales disponibles para explotarla. Para paliar este déficit presentamos aquí una secuencia didáctica pensada para el Español de los negocios de nivel $\mathrm{C} 1$. En el discurso de las ciencias sociales proliferan las metáforas creadas a partir de términos de la lengua común que han adquirido un significado específico en estas disciplinas. Trabajamos con textos de la sección económica de El País y nos fijamos en las metáforas conceptuales subyacentes, que soportan buena parte del peso argumentativo. Tras este entrenamiento, el alumno deberá construir metáforas para debatir sobre el intervencionismo en economía.

Palabras clave: Metáfora, lingüística cognitiva, economía, español de los negocios

\begin{abstract}
As a result of the formulation of the Cognitive Theory of the Metaphor by Lakoff and Johnson (1980) scholars discovered that this literary device could contribute to the development of communicative competence among L2 students. However, there are barely any didactic materials to take advantage of it. With the purpose of filling this gap a didactic sequence for Business Spanish, level C1, is presented. The speech in social sciences is rich in technical terms coming from the assignment of a metaphorical value to words we use in everyday language. Texts from the economic section of the newspaper El País will be used in order to detect the underlying conceptual metaphors that are essential for argumentation. After this training lesson, students must be able to construct metaphors for the discussion on economic interventionism.
\end{abstract}

Keywords: Metaphor, cognitive linguistics, economy, Business Spanish

\footnotetext{
${ }^{1}$ Agradezco a Reyes Llopis García los comentarios que realizó a la secuencia didáctica aquí presentada durante su gestación, pues las actividades que la componen formaron originariamente parte del trabajo de fin de máster «La metáfora como recurso didáctico en el aula de ELE: Una propuesta a partir de la lingüística cognitiva» que ella me tuteló en la Universidad Internacional Menéndez Pelayo en 2014.
} 


\section{Por qué hablar de economía con metáforas: criterios metodológicos seguidos en el diseño de la secuencia didáctica}

La formulación de la teoría cognitiva de la metáfora por parte de Lakoff y Johnson (1980) despertó el interés por el potencial didáctico de esta figura para el desarrollo de la competencia comunicativa en la enseñanza de segundas lenguas. Algunos estudiosos, como Danesi (Acquaroni, 2008: 137) no tardaron en ocuparse de ella. Aun así, lo cierto es que, como señalan Littlemore y Low (2006a: XV) la investigación acerca del lenguaje figurado (y por ende de la metáfora incluida en él) es mínima si se la compara con la existente en otras áreas dentro de la adquisición de segundas lenguas. Los estudios más recientes suelen encontrarse, de forma mayoritaria, en el ámbito anglosajón, firmados por autores que se adscriben a la lingüística cognitiva. Este es el caso de las publicaciones en las que está implicada, bien en solitario o con otros colaboradores, Littlemore (2001, 2006a, 2006b, 2009, 2013) y que consideramos nuestra referencia básica en el presente trabajo. Si la comparamos con las indagaciones en lengua inglesa, la atención a la metáfora dentro del marco de ELE está todavía en la indigencia y seguramente la tesis doctoral de Acquaroni (2008) constituya el trabajo más extenso al respecto. Esta situación nos llevó a realizar una encuesta acerca de cómo estudiantes y profesores de ELE valoraban la metáfora. De los resultados de este estudio (Rivera León, 2016) es deudora la presente contribución, pues quienes respondieron al cuestionario coincidían en que, pese a las posibilidades didácticas de la metáfora, eran escasos los materiales para tratarla en el aula de ELE. Asimismo, llegaron a la conclusión de que cuando esta aparecía en los manuales lo hacía, o bien prisionera del corsé de bellos textos literarios o bien relegada al aburrimiento de interminables listados de modismos y frases hechas.

Nuestra propuesta didáctica está pensada para trabajar de forma específica con la metáfora en el ámbito de EFE, en concreto en un curso de español de los negocios de nivel C1. En el discurso de las ciencias sociales, como la economía, que tiene un carácter muy abstracto, son numerosos los términos técnicos creados a través de la asignación de un valor metafórico a palabras del lenguaje cotidiano. En la secuencia empleamos una noticia y un artículo de opinión para poner de manifiesto que, a pesar de que los textos periodísticos no son un vehículo usual para el tratamiento de la metáfora en el aula de ELE (y así lo mostraban los cuestionarios de nuestro estudio), sí que ofrecen grandes oportunidades para la adquisición de vocabulario y permiten, además, abordar de forma crítica la estructuración y la argumentación del discurso.

La referencia principal para el diseño de la secuencia didáctica es el libro publicado por Littlemore y Low (2006) donde estos estudiosos defienden que el lenguaje figurado desempeña un papel fundamental en todas las subcompetencias que integran el modelo comunicativo de Bachman y que es posible desarrollar materiales que exploten esta potencialidad. Presentamos no solo actividades de reconocimiento de la metáfora, sino también de producción y lo hacemos integrando esta de manera natural en los materiales, al tiempo que trabajamos sobre el léxico o diversas tipologías textuales. Concordamos con Littlemore y Low (2006a: 303-329) en que conviene hacer consciente al alumno de que puede servirse de la metáfora en su proceso de aprendizaje de la lengua extranjera, pero no buscamos de él una atención expresa sobre la distinción entre metáforas conceptuales y lingüísticas que sería más propia de filólogos que de estudiantes de ELE.

Aunque la metáfora presenta interés como herramienta didáctica en todos los niveles de enseñanza de ELE, hemos optado por elaborar una propuesta para un nivel $\mathrm{C} 1$ por varios 
motivos. En primer lugar, porque los cuestionarios de profesores que analizamos en nuestro artículo (Rivera León, 2016) apuntaban a que era en los niveles altos donde la metáfora se abordaba de forma más habitual y no es nuestro propósito alterar de manera radical los hábitos de trabajo de los docentes. Nos conformamos con contribuir mínimamente a que descubran en la metáfora aspectos que les pudieran haber pasado desapercibidos. En segundo término, porque algunos estudios (Littlemore et al., 2013: 1) han demostrado que la densidad metafórica de las producciones de los alumnos y de los textos con los que trabajan, aumenta exponencialmente de los niveles A2 a C2. Así, encontrábamos mayores oportunidades para la creación de materiales en los niveles altos. Por último, destaca MacArthur (2010: 155) que la metáfora introduce frescura y por tanto despierta la motivación en niveles donde los estudiantes sienten que su aprendizaje se ha estancado y precisan nuevos estímulos.

En consonancia con lo defendido por el Marco Común Europeo de Referencia para las lenguas (MCER) y el Plan Curricular del Instituto Cervantes (PCIC) elaborado a partir de sus recomendaciones, se adopta un enfoque orientado a la acción y centrado en el alumno. Nuestra propuesta exige una toma de conciencia por parte de este último y promueve su actitud reflexiva. Una buena muestra de ello es la actividad 5, «La economía a debate», que hemos creado inspirándonos en una propuesta similar que Littlemore llevó al aula en un contexto universitario con profesores japoneses que cursaban un máster en enseñanza del inglés como lengua extranjera (Littlemore y Low, 2006a: 121-126). Partimos de un enfoque cognitivo y por tanto hemos de asumir que el estudiante participa de forma activa en su proceso de aprendizaje utilizando diversas estrategias mentales con el fin de organizar el sistema lingüístico que va a aprender. Asimismo, y de acuerdo con los principios del interaccionismo social, hemos cuidado las técnicas de agrupación, procurando introducir dinamismo en el aula y dar cabida a diversos estilos de aprendizaje.

\section{Guía del profesor}

\subsection{Nivel: $\mathrm{C} 1$}

\subsection{Destinatarios}

La secuencia didáctica está pensada para ser llevada al aula en un curso de Español de los negocios. En cuanto al alumnado, dado el carácter especializado de los textos que se utilizan, lo ideal es que se trate de estudiantes universitarios de las ramas de Economía o Administración y Dirección de Empresas. También los profesionales del mundo de la empresa, los ejecutivos con intereses en España o Latinoamérica y los trabajadores diplomáticos podrían sacar buen provecho de estos materiales.

\subsection{Objetivos}

- Ser capaz de comprender noticias y artículos de opinión extraídos de la sección económica de un periódico.

- Ser capaz de reconocer metáforas lingüísticas y conceptuales en una noticia económica y en un artículo de opinión de carácter argumentativo.

- Ser capaz de resumir el contenido de una noticia económica utilizando una imagen metafórica.

- Tomar conciencia de la posibilidad de usar de manera estratégica la metáfora para articular textos argumentativos y mejorar así la expresión escrita. 
- Ser capaz de construir argumentos a partir de una imagen metafórica y usarlos en un debate sobre un tema económico complejo.

- En relación con el componente sociocultural, aproximarse a la realidad de la generación de españoles nacidos en los años 80 a partir de la visión irónica que un cómic ofrece de ella.

- Ser capaz de llegar a acuerdos con los compañeros para distribuir distintos roles en la realización óptima de una tarea colaborativa.

- Ser capaz de escribir un artículo de opinión sobre economía.

\subsection{Destrezas lingüísticas implicadas}

Interacción oral, comprensión escrita, expresión escrita.

\subsection{Desarrollo}

La secuencia didáctica que presentamos está integrada por seis actividades que pasamos a detallar a continuación antes de proporcionar el material para el alumno.

La primera actividad tiene como objetivo presentar el tema en el que va a estar centrada la secuencia didáctica, que no es otro que la economía. Se proporcionan cuatro listados de palabras que se han extraído de los textos con los que se trabajará en las actividades 2 y 3: una noticia y un artículo de opinión publicados ambos en la sección económica de $E l$ País. Aunque todos los términos se han usado en un contexto económico, lo cierto es que parecen pertenecer a otros campos léxicos, en concreto: (1) enfermedad, medicina; (2) guerra, enfrentamientos; (3) máquinas, automóviles; (4) agua, experimentos de física. Por ello se les pide a los alumnos que aventuren de qué podríamos estar hablando en cada caso al usar esas palabras. Cuando posteriormente descubran que todas ellas formaban parte de textos económicos, sin duda se darán cuenta de que los lenguajes técnicos de las ciencias sociales están impregnados de términos del lenguaje cotidiano cuyo significado originario no coincidía exactamente con el que han terminado adquiriendo en esa disciplina. La actividad puede hacerse individualmente o por parejas.

La actividad 2 es, sobre todo, de comprensión lectora. Sin embargo, esta no se aborda solo mediante las preguntas habituales sobre el contenido del texto, sino que se añaden además ejercicios que buscan profundizar en el componente metafórico de la noticia. Asimismo, esta actividad sirve de introducción para el tema que se tratará ya hasta el final de la secuencia: el dilema sobre la conveniencia o no del intervencionismo en economía. Es posible tanto el trabajo individual como en parejas o pequeños grupos, a lo cual se añade una puesta en común al final.

En la actividad 3 que, al igual que la anterior, es fundamentalmente de comprensión lectora, se plantea un primer ejercicio previo a la lectura con el que se pretende contextualizar desde el título del artículo hasta el símil literario que le sirve de eje articulador. Este consiste en comparar la situación de abandono que viven los países del sur de Europa, a la espera de que sus vecinos del norte muevan ficha para resolver el asfixiante problema de la deuda, con la actitud vital de los personajes de Esperando a Godot de Samuel Beckett. Mario Draghi, presidente del Banco Central Europeo, es el Godot de esta nueva tragedia del absurdo en el viejo continente. Tras la lectura del artículo de opinión se presenta un primer bloque de preguntas de comprensión sobre su contenido. En segundo lugar, hay otro conjunto de cuestiones que inciden ya más directamente en la presencia de metáforas en el texto, tanto de tipo lingüístico como conceptual. En cuanto 
a las técnicas de agrupación, hay posibilidades tanto para el trabajo individual como en parejas o pequeños grupos con una puesta en común al final.

Con la actividad 4 se pretende preparar a los alumnos para que sean capaces de pensar sobre cuestiones complejas y abstractas sirviéndose de imágenes que permitan analogías clarificadoras. Hemos escogido unas viñetas de un cómic de Aleix Saló en las que se establece un símil entre un coche y la generación de los jóvenes de hoy nacidos en los años 80, entre los que él mismo se cuenta. En la siguiente actividad les pediremos a los alumnos que piensen en la economía como si se tratara de un coche a fin de que puedan crear, a partir de esa imagen, argumentos a favor o en contra del intervencionismo. Trabajando ahora con el cómic los estamos preparando para esa tarea. Hay que tener en cuenta que será necesario ofrecer información acerca del contexto de las viñetas, pues se cita, por ejemplo, a los «adalides progres de la Transición». También habrá que detenerse en resolver dudas sobre el vocabulario coloquial («irse al carajo»). Aunque se plantea un ejercicio sencillo de comprensión, seguramente convenga ir más allá de él en las explicaciones.

La actividad 5 recoge todo lo visto hasta el momento en la secuencia didáctica para organizar un debate sobre el tema central de los textos trabajados: el intervencionismo en economía. Para construir argumentos en uno u otro sentido los estudiantes tendrán que pensar en la economía como si fuera un coche. Puesto que llevar a cabo con éxito un debate de estas características resulta muy complejo, se ha optado por pautarlo y controlarlo lo máximo posible. Así, se divide la clase en dos grupos y se le asigna a cada uno una posición que defender en el debate. En cada grupo ha de haber un secretario que anote los argumentos y el resto de miembros se tendrá que turnar para exponerlos.

La última actividad de la secuencia didáctica está pensada como una prolongación del debate previo y por tanto confluye también aquí todo lo que se ha tratado anteriormente. De manera individual, los alumnos deberán redactar un texto en el que expresen su opinión acerca del intervencionismo en economía. A su escrito tendrán que adjuntar un pequeño comentario en el que especifiquen qué expresiones metafóricas del tipo de las estudiadas en la lección han empleado. Si se prefiere, esta actividad podría también plantearse como de escritura colaborativa, solo que, al haber trabajado ya en grupo en el debate, se ha optado aquí por un ejercicio individual.

\subsection{Solucionario}

\section{Actividad 1: ¿De qué estamos hablando?}

1. enfermedad, medicina; 2. guerra, enfrentamientos; 3 . máquinas, automóviles; 4. agua, experimentos de física.

\section{Actividad 2: Una medicina de muchos dólares}

Apartado $a$ : 1 V, 2 F, 3 F, 4 V, 5 V, 6 F

Apartado $b$, cuestión 3: «La economía es un paciente» (opción $b$ ), es la solución más obvia, aunque puede admitirse alguna otra siempre que el alumno lo justifique adecuadamente.

\section{Actividad 3: ¿Pero esto no iba de literatura?}

Apartado $c$ : 
Cuestión 1: Los que están siempre esperando a que llegue Godot son los Gobiernos del norte de Europa que no se implican directamente en la búsqueda de soluciones al problema de la deuda que afecta básicamente al sur de Europa. Los espectadores de la obra son los ciudadanos del sur de Europa que sufren directamente la crisis sin que sus mandatorios hagan nada desde los órganos gubernamentales de la Unión Europea.

Cuestión 5: La metáfora fundamental es en este caso la de la economía como guerra.

\section{Actividad 4: ¡Qué coche más raro!}

$1 \mathrm{~F}, 2 \mathrm{~V}, 3 \mathrm{~V}, 4 \mathrm{~F}, 5 \mathrm{~F}$

\section{Bibliografía}

ACQUARONI, R. (2008). La incorporación de la competencia metafórica (CM) a la enseñanza-aprendizaje del español como segunda lengua (L2) a través de un taller de escritura creativa: estudio experimental. Tesis doctoral. Madrid: Universidad Complutense.

GaricAno, L. (21/06/2013). «Esperando a Godot-Draghi». El País. Recuperado el 29 de octubre de 2014 de: http://economia.elpais.com/economia/2013/04/19/actualidad/1366365953_382283.html

JiMÉNEZ, V. (2014, 29 de octubre). «La Reserva Federal de EE UU pone fin a seis años de estímulos masivos». El País. Recuperado el 29 de octubre de 2014 de: http://economia.elpais.com/economia/2014/10/29/actualidad/1414607394_203338.html

LAKoff, G. \& M. Johnson (1980). Metaphors We Live by. Chicago: University of Chicago. [Trad. cast.: Id.: Metáforas de la vida cotidiana, trad. de C. González Marín. Madrid: Cátedra, 2012. (1 ${ }^{\mathrm{a}}$ ed. 1986)].

Littlemore, J. (2001). «Metaphoric Competence: A Language Learning Strength of Students with a Holistic Cognitive Style?». TESOL Quarterly, 35/3, 459-491.

LitTlemore, J. \& G. LOW (2006a). Figurative Thinking and Foreign Language Learning. Basingstoke: Palgrave MacMillan.

Littlemore, J. \& G. Low (2006b). «Metaphoric Competence, Second Language Learning, and Communicative Language Ability». Applied Linguistics, 27/2, 268-294. DOI: $10.1093 /$ applin/am1004

LitTlemore, J. (2009). Applying Cognitive Linguistics to Second Language Learning and Teaching. Basingstoke: Palgrave MacMillan.

LitTlemore, J., T. Krennmayer, J. TuRner \& S. TuRner (2013). «An Investigation into Metaphor Use at Different Levels of Second Language Writing». Applied Linguistics, 1-29. DOI: 10.1093/applin/amt004

MacArthur, F. (2010). «Metaphorical Competence in EFL: Where Are We and Where Should We Be Going? A View from the Language Classroom». En: J. Littlemore \& C. Juchem-Grundmann (eds.), Applied Cognitive Linguistics in Second Language Learning and Teaching. AILA Review, 23/1, 155-173. DOI: 10.1075/aila.23.09mac

RIVERA LEÓN, L. (2016). «La metáfora como recurso didáctico en el aula de ELE: Un estudio a partir de la lingüística cognitiva». Boletín de la Asociación para la Enseñanza del Español como Lengua Extranjera (ASELE), 54, 13-46. 
SAló, A. (2014). Hijos de los 80. La Generación Burbuja, trad. cast. de C. Mayor. Barcelona: Penguin Random House.

VV.AA. (2002). Marco común europeo de referencia para las lenguas: aprendizaje, enseñanza, evaluación, (MCER), trad. cast. Madrid: MECD/Anaya.

VV.AA. (2006). Plan curricular del Instituto Cervantes. Niveles de referencia para el español, 3 vols. Madrid: Biblioteca Nueva. 
Anexos. Material para el alumno

\section{Actividad 1: ¿De qué estamos hablando?}

A continuación, tienes cuatro listas de palabras o de expresiones. Di de qué podríamos estar hablando al usarlas en cada uno de los cuatro casos con un concepto representativo. Usa el diccionario si no conoces alguna palabra.

1. Inyectar, adicción, recuperación robusta, recuperación lenta, paciente, sacudidas, síntomas, adolecer de una cierta debilidad, animarse, seguir renqueante, deterioro, perecer, indoloro.

2. Obstáculos, presión, retirada, mantener amarrado, pánico, brusquedad, lanzar tres oleadas, desolador, horroroso, presionar, amenazar, intervenir con fuerza, explosión inmediata e incontrolada, contracción, riesgo, lanzarse, impacto, atrapado, poner en marcha una respuesta agresiva, inacción, ejecutar, destruido, reacción, forzar, guerra de trincheras, perecer.

3. Motor, bombear, desenganche, calibre, acelerar, apalancamiento, encadenar, atascado, poner en marcha.

4. Inundar, liquidez, bombear, volátil, inyectar, sólido, presión, cerrar el grifo, oleada, cerrar la manguera, ahogado.

\section{Actividad 2: Una medicina de muchos dólares}

E1 29 de octubre de 2014 la Reserva Federal (Fed) de Estados Unidos daba una importante noticia para la economía norteamericana y, en consecuencia, para la economía mundial. Lee cómo el diario El País recogió la información y realiza las actividades que se proponen en los distintos apartados.

\section{La Reserva Federal de EEUU pone fin a seis años de estímulos masivos}

El banco central anuncia que mantendrá los tipos de interés en el $0 \%$ durante «un tiempo considerable»

Acaba una época, un experimento único de una de las peores crisis del capitalismo. La Reserva Federal ha puesto fin este miércoles al programa de compra masiva de activos financieros y préstamos hipotecarios para inundar de liquidez los mercados y estimular la economía de Estados Unidos. Noviembre será el primer mes de los últimos 37 en que la Fed no comprará deuda, lo que supone dejar la primera economía del planeta sin el motor que empezó a bombear dinero en 2008, tras la hecatombe de Lehman Brothers.

El comunicado del banco afirma que los tipos de interés seguirán en el $0 \%$ durante un periodo de tiempo «considerable», probablemente hasta mediados de 2015 (la Fed mantendrá un balance de activos de 4,5 billones de dólares), siempre y cuando se confirmen los indicadores actuales y la inflación, controlada en el $1,7 \%$, siga dando margen.

El tono general del documento es positivo, con el objetivo de tranquilizar a los mercados, algo volátiles los últimos días. Tenían motivos para ello. Está por ver si los inversores superan su adicción a los más de tres billones y medio de dólares inyectados en estos años y si la recuperación de EEUU es tan robusta como para navegar sola. Janet Yellen, presidenta de la Fed, cree llegado el momento del desenganche.

El banco central certifica una «expansión moderada» de la economía con un «sólido» ritmo de creación de empleo. En este sentido, y en contraste con anteriores comunicados, destaca la «gradual reducción de los obstáculos» para la contratación. Sin embargo, precisa que la presión inflacionista es a la baja y que el mercado inmobiliario muestra una «recuperación lenta», lo que justificaría un margen de tiempo antes de subir el precio del dinero.

Acertar en el momento y en el calibre de la subida de los tipos es uno de los puntos más peliagudos que debe afrontar la Fed. Y las presiones no ayudan. Los dueños de bonos, poderosos fondos, firmas financieras y magnates de todo tipo quieren unos tipos que mantengan 
amarrada la inflación, para así no ver depreciados sus títulos.

En junio de 2013, la simple sugerencia de una retirada de estímulos por parte del entonces jefe del banco central de EEUU, Ben Bernanke, provocó el pánico en las Bolsas. De ahí que el cierre del grifo haya sido progresivo, para que el paciente no acusara el cambio con brusquedad. La retirada escalonada ha permitido que los mercados asumieran este miércoles la noticia sin sacudidas.

La operación de estímulos masivos comenzó en 2008. Tras la caída de Lehman Brothers y la confirmación de una de las peores crisis en la historia del capitalismo, el banco central de EEUU bajó sus tipos de interés casi a cero para intentar cebar los motores de la economía. No fue suficiente y tuvo que lanzar tres oleadas de compra de deuda en el mercado (2008, 2010 y 2012). En la última de ellas, el compromiso fue cerrar la manguera en cuanto la economía ofreciera síntomas de estabilización y un crecimiento razonable. El momento ha llegado, aunque con matices.

Desde 2012, el desempleo ha bajado de un $8,1 \%$ al $5,9 \%$ en septiembre. La previsión de crecimiento para este año es del 1,7\%, y del 3\% para 2015. Los indicadores de producción están en positivo. La recuperación, por lo tanto, es un hecho. Los analistas advierten, sin embargo, que adolece de una cierta debilidad. Un ejemplo son los salarios, que están estancados. El mercado inmobiliario tampoco las tiene todas consigo. La construcción y los precios se han animado, pero el crédito sigue renqueante.

Texto adaptado a partir de: Vicente Jiménez, «La Reserva Federal de EEUU pone fin a seis años de estímulos masivos», noticia publicada en El País el 29/10/2014.

a) Indica en cada caso si las afirmaciones son verdaderas o falsas:

\begin{tabular}{|c|c|c|}
\hline & $\mathrm{V}$ & \\
\hline 1. En noviembre la Reserva Federal ya no comprará deuda. & & \\
\hline 2. La subida de los tipos de interés será inmediata. & & \\
\hline $\begin{array}{l}\text { 3. No hay dudas de que la economía norteamericana podrá seguir adelante } \\
\text { sin la ayuda de la Reserva Federal. }\end{array}$ & & \\
\hline $\begin{array}{l}\text { 4. El banco central considera que hay indicadores positivos en la economía } \\
\text { norteamericana, pero que hay que mantener la cautela. }\end{array}$ & & \\
\hline 5. Los estímulos a la economía se han ido eliminando progresivamente. & & \\
\hline $\begin{array}{l}\text { 6. La primera decisión que tomó el banco central para darle empuje a la } \\
\text { economía fue comprar deuda. }\end{array}$ & & \\
\hline
\end{tabular}

b) A partir del texto, responde a las siguientes preguntas:

1. ¿Recuerdas las palabras y las expresiones de la actividad 1? ¿Puedes encontrar algunas de ellas en el texto? Señálalas.

2. En el texto se habla de tranquilizar a los mercados y se dice que estos tenían motivos para estar volátiles, o sea, inestables. Pero, ¿no son en sentido estricto las personas las que necesitan que las tranquilicen y las que pueden tener motivos para determinadas cosas? Busca en el texto al menos tres ocasiones más en las que se atribuyan cualidades, sentimientos o comportamientos humanos a objetos o a conceptos abstractos, como lo es la economía.

3. ¿Cuál de las siguientes cuatro metáforas crees que es la predominante en el texto? Justifica tu respuesta y di qué relación puede haber entre esa imagen y la noticia que se transmite.
a) La economía es agua
b) La economía es un paciente
c) La economía es una máquina
d) La economía es guerra 


\section{Actividad 3: ¿Pero esto no iba de literatura?}

a) En la actividad n 2 hemos visto la noticia de cómo el 29 de octubre de 2014 la Reserva Federal de Estados Unidos decidió interrumpir los estímulos a la economía norteamericana que había venido manteniendo desde el inicio de la crisis en 2008. Precisamente en abril de 2013 un economista español, Luis Garicano, escribía en el periódico El País un artículo de opinión destacando las consecuencias positivas que las intervenciones de la Reserva Federal habían tenido para la economía de Estados Unidos y pidiendo a los gobiernos del norte de Europa que asumieran la necesidad de actuar sobre la deuda de las economías de Europa del sur, las más castigadas en el continente por la crisis. Vamos a leer ese texto, pero contesta antes a las siguientes preguntas:

1. Esperando a Godot es una obra de teatro de Samuel Beckett. ¿Qué sabes de ella?

2. ¿Sabes quién es Mario Draghi?

3. Con la información que ya tienes sobre Esperando a Godot y leyendo solo el titular del artículo, ¿te parece que su autor manifestará mucha confianza en el papel del Banco Central Europeo (BCE) que preside Mario Draghi? Justifica tu respuesta.

\section{Esperando a Godot - Draghi}

Marzo ha sido un mes desolador para la eurozona, incluida España. En todo el conjunto de la zona euro, la tasa de deterioro del índice de gestión de compras se ha acelerado por segundo mes, encadenando 19 meses de caída de la producción. El desempleo de la zona euro subió en los últimos 12 meses del 10,9\% al 12\%. Estos horrorosos indicadores adelantados muestran que la economía europea se sigue deteriorando. Lo que estamos haciendo no funciona, como el FMI ha dejado claro recientemente.

El Gobierno español muestra ya públicamente su preocupación y su descontento con la política del BCE. El ministro Luis de Guindos ha presionado públicamente al $\mathrm{BCE}$ en la reunión del Eurogrupo en Dublín para que actúe, y Rajoy, por primera vez, hizo lo mismo en varias declaraciones recientes. El Gobierno siente que de esta no salimos solos.

Las palabras mágicas de Mario Draghi en Londres en julio de 2012, amenazando con una intervención decidida del BCE, sirvieron para parar el pánico en los mercados. Hoy pocos apuestan contra la deuda pública de España e Italia. Imaginan que si las cosas se ponen suficientemente mal, el BCE intervendrá con fuerza a favor de estos países.

Pero si bien estas palabras sirvieron para evitar una explosión inmediata e incontrolada, no son en absoluto suficientes para poner en marcha las débiles y extremadamente apalancadas 2 economías de la periferia de Europa.

\footnotetext{
2 «Apalancarse» es sinónimo de «endeudarse» en economía. Por ello, «apalancado» es
}

El problema es que hemos consumido ya lo que produciremos en el futuro, generando elevadas deudas. Ahora tenemos que devolver estos préstamos, desapalancarnos. Esto sería más o menos indoloro con crecimiento económico (como en la Europa de la posguerra), y relativamente indoloro también en un contexto inflacionista, en el que las deudas pierden valor real con el tiempo.

Pero, a falta de inflación y de crecimiento, el proceso de desapalancamiento requiere una fuerte contracción del consumo para generar suficiente excedente. El problema es que la caída de consumo de una familia (los cafés que se dejan de tomar) son los ingresos de otra (los ingresos del dueño del bar) -cuando caen todos a la vez no se produce ningún incremento del excedente y las economías entran en una espiral deflacionista, ahogadas por el peso de la deuda.

La Reserva Federal (Fed) de EEUU y el Banco de Japón han entendido este riesgo perfectamente y se han lanzado por una senda antes inconcebible de expansión monetaria, tratando de incrementar las expectativas de inflación. La Fed ha anunciado que mantendrá los tipos a cero hasta que no baje sustancialmente el desempleo. El Banco de Japón multiplicará la base monetaria (es decir, la moneda y depósitos) por dos, tratando de elevar las expectativas de inflación y así reducir los tipos de interés reales.

Desgraciadamente, ni el BCE va a ser capaz de tomar estas decisiones tan agresivas, ni las

«endeudado» y «desapalancarse» es «desendeudarse». 
decisiones que ya está tomando tienen el impacto que pueden tener en EEUU o Japón. Es decir, hay obstáculos tanto institucionales como económicos que impiden al BCE tener un impacto directo sobre la economía europea.

El primer obstáculo es institucional. El marco europeo de política económica, en el que estamos atrapados desde 2010, no permite a Europa las agresivas respuestas que han puesto en marcha japoneses y estadounidenses. Por otro lado, tampoco las (insuficientes) decisiones que el BCE está pudiendo tomar tienen el impacto sobre los hogares y las empresas que pueden tener en EEUU o Japón, porque, dado el atascado sistema financiero de la periferia, las reducciones del coste de financiación de Estados y bancos no llegan ni a familias, ni a empresas.

En definitiva, el BCE ni está, ni se le espera. Como los personajes de la obra de Beckett, nuestros políticos cubren el silencio y su inacción en desorientada conversación («no hacer nada... es lo más seguro», dice uno de los caracteres) y preguntándose qué pasará cuando por fin llegue Godot-Draghi. Pero los espectadores nos tememos que, cuando acabe la obra, Godot no habrá llegado.

¿Qué hacer? Como hemos dicho repetidamente, España debe hacer su parte, y esto supone poner en marcha un plan de reactivación y reformas que solo un Gobierno como el actual, con mayoría absoluta, sería capaz de ejecutar. Y Europa debe hacer la suya: ser capaz de financiar un verdadero Plan Marshall para la destruida Europa del Sur, entendiendo la necesidad de compartir las deudas heredadas de un diseño del euro que fue erróneo y es responsable directo de la enorme burbuja y la nula reacción frente a ella. Desgraciadamente, los Gobiernos del norte insisten en que no es su problema, tratando de forzar la mano del BCE.

Y así, en esta guerra de trincheras entre el BCE y los Gobiernos del norte de Europa, mientras los del sur esperan a Godot, siguen pereciendo las esperanzas de innumerables familias europeas.

Texto adaptado a partir de: Luis Garicano, «Esperando a Godot-Draghi», artículo de opinión publicado en El País el 21/04/2013.

b) Una vez leído el texto, responde a las siguientes preguntas:

1. ¿Cuál es el estado de la economía europea según el texto? Descríbelo brevemente.

2. ¿Cuál es la actitud del gobierno de España, que preside Rajoy, ante la política del Banco Central Europeo?

3. ¿Qué efecto tuvo el anuncio de Mario Draghi hecho en Londres en julio de 2012?

4. ¿Por qué la deuda se ha convertido en un gran problema precisamente en el contexto económico que se describe?

5. ¿Qué tipo de política monetaria han llevado a cabo la Reserva Federal (Fed) de Estados Unidos y el Banco de Japón?

6. ¿Puede el Banco Central Europeo imitar la política monetaria de la Reserva Federal de Estados Unidos y el Banco de Japón? ¿Por qué?

7. ¿Qué debería hacer España para combatir la crisis según el autor?

8. ¿Qué debería hacer Europa en la situación que se describe?

c) Como hemos visto, en el artículo se establece, ya desde el mismo título, un paralelismo entre la obra de teatro Esperando a Godot y los protagonistas de la crisis europea. Teniendo esto en cuenta, responde a las siguientes preguntas:

1. Si Mario Draghi equivale a Godot, ¿quiénes serían, en el escenario europeo, los otros protagonistas de la obra, los que están siempre esperando a que Godot llegue? ¿Y quiénes serían los espectadores de la obra? Para responder, fíjate sobre todo en el antepenúltimo párrafo del texto.

2. Después de haber leído el texto, ¿crees que el autor del artículo tiene mucha confianza en que el Banco Central Europeo presidido por Mario Draghi y los gobiernos del norte 
de Europa pongan en marcha políticas económicas que supongan una ayuda para los países del sur del continente?

3. ¿Qué te ha parecido la construcción del artículo a partir de un paralelismo con una obra literaria como Esperando a Godot? ¿Crees que es un recurso habitual para estructurar textos argumentativos como este? ¿Tú lo utilizas cuando escribes en tu propia lengua?

4. ¿Recuerdas las palabras y expresiones que vimos en la actividad 1 de la lección? Muchas de ellas están tomadas de este texto. Búscalas.

5. De acuerdo con lo que has encontrado, ¿cuál es el campo temático, de los recogidos en la actividad 1, del que más palabras y expresiones se utilizan en el texto? ¿Guarda esto relación con la idea que quiere transmitir el artículo?

6. Tanto en la noticia analizada en la actividad 2 como en el artículo de opinión de Luis Garicano hemos visto que, de manera habitual, para hablar de economía se emplean muchas palabras procedentes de distintos campos léxicos que han extendido de alguna manera su sentido más inmediato y que ya forman parte habitual del lenguaje usado para hablar de economía. Sin embargo, arrastran consigo su sentido primero, de manera que lo que se transmite usando en un texto sobre economía muchos términos que se relacionen con la enfermedad es muy distinto de lo que se comunica empleando palabras que tengan que ver con la guerra. ¿Crees que ser consciente de estos usos puede ayudarte a argumentar mejor cuando escribes? Comenta tus opiniones con un par de compañeros.

\section{Actividad 4: ¡Qué coche más raro!}

En su cómic Hijos de los 80. La generación burbuja, Aleix Saló hace un retrato de los jóvenes que, como él mismo, nacieron en España en la década de los 80 y que cuando escribió esta obra (2009) estaban, por tanto, en la veintena. En un momento del libro plantea un paralelismo entre los jóvenes de esta generación y un automóvil. Observa las viñetas y realiza el ejercicio propuesto. 

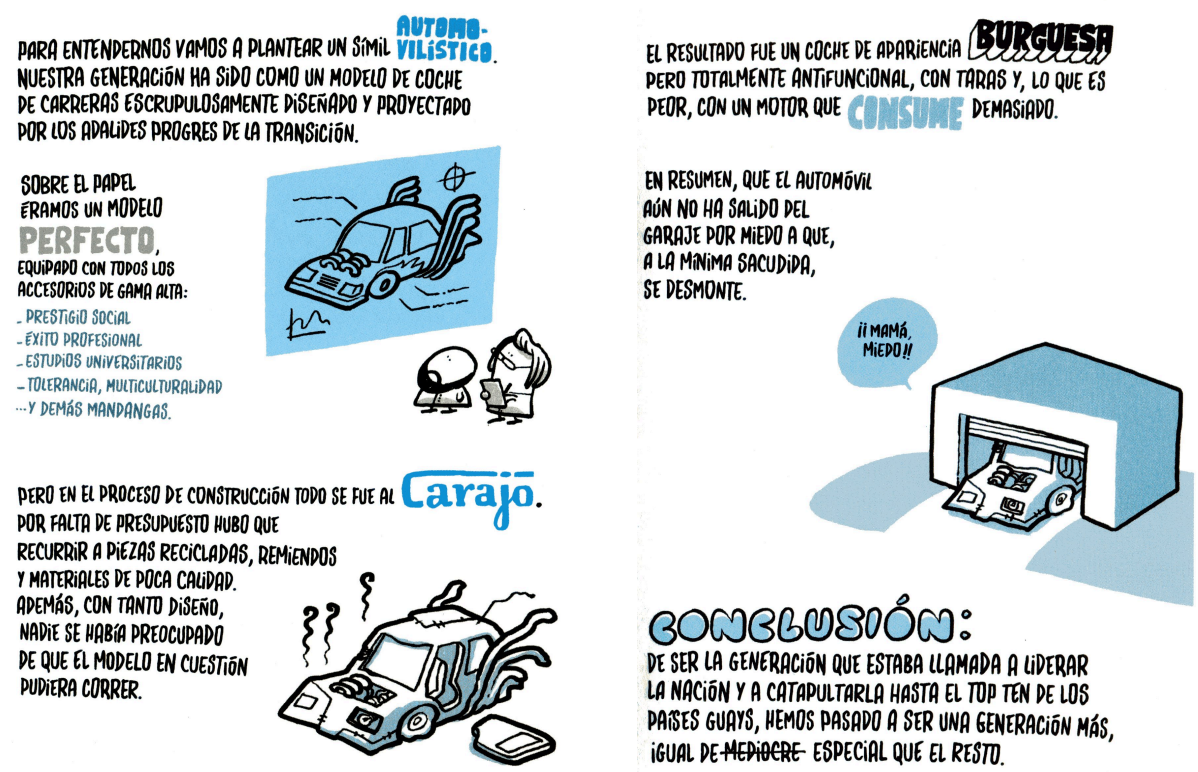

Imagen 1. Viñeta tomada de: Aleix Saló (2014), Hijos de los 80. La generación burbuja. Barcelona: Penguin Random House, pp. 16-17.

Decide si las siguientes afirmaciones son verdaderas o falsas:

\begin{tabular}{l|l|l} 
& $\mathrm{V}$ & $\mathrm{F}$ \\
\hline 1. La juventud española tiene poca formación académica. & & \\
\hline $\begin{array}{l}\text { 2. Los jóvenes españoles tienen dificultades para andar solos poniendo } \\
\text { en práctica todo lo que saben. }\end{array}$ & & \\
\hline 3. Los jóvenes españoles son consumistas. & & \\
\hline $\begin{array}{l}\text { 4. Los jóvenes españoles son valientes. } \\
\text { 5. La juventud española será capaz de alcanzar grandes logros para su } \\
\text { país }\end{array}$ & & \\
\hline
\end{tabular}

\section{Actividad 5: ¡La economía a debate!}

Al trabajar con los dos textos de las actividades 2 y 3 , hemos aprendido que cuando hablamos de economía empleamos muchas palabras y expresiones procedentes de otros campos léxicos como el de la guerra o la medicina. Así, por ejemplo, era posible referirse a la economía como una máquina o como un coche, que acelera, calienta sus motores, avanza a buen ritmo, etc. Por otro lado, hemos visto en la actividad 4 cómo Aleix Saló comparaba en sus viñetas a la juventud española con un coche.

En esta actividad vamos a jugar con la imagen del coche para aplicarla a la economía y crear un debate en clase que llevará por título «¿A favor o en contra del intervencionismo en economía?». Nos dividiremos en dos grupos y, por sorteo, a uno de ellos le tocará estar a favor del intervencionismo y al otro en contra. Cada grupo tendrá que buscar argumentos para defender su postura, pero pensando en la economía como si fuera un coche, de manera parecida a como lo hace Aleix Saló en sus viñetas con los jóvenes españoles. 
La noticia de la actividad 3 relataba cómo la Reserva Federal había intervenido en la economía de Estados Unidos y los efectos que podía tener que dejara de hacerlo. En el artículo con el que hemos trabajado en la actividad 4, su autor, Luis Garicano, pedía a los gobiernos del norte de Europa que adoptaran medidas para actuar sobre la deuda. De ambos textos podéis extraer ideas para crear argumentos para el debate y además tenéis abajo un ejemplo para cada posición que os puede ayudar.

En cada grupo deberá haber un secretario que anote los argumentos y tendréis que decidir quién expone cada uno. Es necesario que hablen distintas personas. No puede haber un solo portavoz. Además, cuando el grupo contrario dé un argumento, tendréis tiempo para responder a lo que digan a partir de lo que hayáis escrito o de una réplica que se le ocurra en ese momento a algún miembro del grupo. Podéis acudir al profesor en cualquier momento, pero sobre todo os será de mucha ayuda al comienzo de la actividad, cuando estéis pensando en los argumentos.

¿Preparados, listos...? ¡En marcha! ¡Buen debate y que gane el mejor!

\section{Ejemplos:}

\section{A favor del intervencionismo:}

Hay coches pequeños y viejos (economías de países más desfavorecidos) que van a un ritmo muy lento, pero que no suponen ningún riesgo para otros vehículos o peatones y hay en cambio coches de carreras (economías muy potentes) que quieren correr sin importarles a quién atropellan. Por eso es necesario que la política, como un guardia de tráfico, establezca algunas normas básicas y fije límites de velocidad.

\section{En contra del intervencionismo:}

Nada garantiza que el mecánico (la política) empeñado en revisar los motores de un buen coche de carreras (economías fuertes) vaya a ser capaz de plantear mejoras a un mecanismo diseñado por los ingenieros (economistas expertos) para funcionar por sí mismo, sin ninguna intervención externa que incluso podría estropearlo.

\section{Actividad 6: ¿Y tú cómo lo ves?}

Escribe un pequeño texto en el que expliques tu postura con respecto al intervencionismo en economía. Puedes contextualizarlo en el panorama económico actual a partir de lo que has aprendido trabajando con la noticia y el artículo de opinión en las actividades 2 y 3. Lo más importante es que tienes que usar léxico que, como «inyección de liquidez»o «la amenaza de una tercera recesión», provenga de otros campos y se aplique de manera habitual a la economía. Si quieres, puedes construir todo tu discurso a partir de una imagen o comparación, como hace Luis Garicano cuando recurre a Esperando a Godot. Al texto tienes que adjuntar un pequeño comentario en el que especifiques cuáles son las palabras o expresiones procedentes de otros ámbitos léxicos que has empleado en tu escrito. (Extensión aproximada del texto: 500 palabras) 